\title{
Extranasopharyngeal Angiofibroma from Nasal Vestibule: A Rare Presentation
}

\author{
Sharanabasappa Rudragouda Malipatil
}

\section{ABSTRACT}

The term extranasopharyngeal angiofibroma has been applied to vascular, fibrous nodules occurring outside the nasopharynx. These rare benign neoplasms characterized by a different biological history and clinical features with respect to nasopharyngeal tumors and, for these reasons, they should be regarded as atypical clinical entity.

A 60-year-old male presented to us with a mass in the left nasal cavity since last 1 year. Examination revealed an irregular, pinkish mass arising from the lateral aspect of left nasal vestibule. The mass was excised and sent for histopathological reporting which confirmed angiofibroma. This case is reported for its atypicality being the rarest site of origin with no case reported so far in medical literature.

Keywords: Nasal vestibule, Angiofibroma, Extranasopharyngeal angiofibroma.

How to cite this article: Malipatil SR. Extranasopharyngeal Angiofibroma from Nasal Vestibule: A Rare Presentation. Int J Otorhinolaryngol Clin 2013;5(3):169-172.

Source of support: Nil

Conflict of interest: None declared

\section{INTRODUCTION}

In 1980, De Vincentiis and Pinelli ${ }^{1}$ reviewed a series of 704 cases of angiofibroma and found that 13 cases manifested outside the nasopharynx, thus suggesting that extranasopharyngeal localization of this tumor is a possible, although rare occurrence. Since then, numerous studies have reported cases of angiofibroma localized in unusual sites. ${ }^{2-5}$ A recent review of the literature reported a total of 65 patients with atypical localizations of nasopharyngeal angiofibromas, ${ }^{6}$ the maxillary sinus being the most commonly involved site. ${ }^{4}$ Nasal septum represents an extremely rare localization with only six cases having been reported in the international literature to date. ${ }^{4,6,7}$ Besides the different location, typical clinical characteristics of extranasopharyngeal angiofibromas, such as, symptoms, age, sex, do not conform to a great extent with that of nasopharyngeal angiofibromas. This fact has led to doubt as to whether extranasopharyngeal angiofibromas, though structurally similar, should be considered as being different from nasopharyngeal angiofibroma. ${ }^{8}$ Recently, Celik et $\mathrm{al}^{9}$ also proposed that patients who have different characteristics other than the classical angiofibromas, should be referred to as 'atypical angiofibroma'. Due to these different features, extranasopharyngeal angiofibromas can present a diagnostic challenge and a meticulous evaluation with a high index of suspicion is essential in establishing the correct diagnosis and treatment. Herein, a rare case is reported of extranasopharyngeal angiofibroma originating from the lateral aspect of left nasal vestibule which is the rarest site with no case being reported so far in medical literature and the idea is, therefore, supported that it must be regarded as atypical.

\section{CASE REPORT}

A 60-year-old male presented at the Otolaryngology Department of BLDEU's Hospital with a 1-year history of mass in the left nasal cavity (Fig. 1). It was incidious in onset, started as a pea-sized mass and progressive in nature to attain the present size. There was no history of bleeding from nose and recurrent cold. The patient had no previous history of trauma or infection. There was no history suggestive of allergy. On ENT examination, anterior rhinoscopy showed a minimal nasal septum deviation on the right side with mass filling the whole of left vestibule (Fig. 2). While nasal endoscopy revealed a pinkish mass $3 \times 4 \mathrm{~cm}$ adhering to the lateral aspect of left nasal vestibule and filling the anterior aspect of nasal cavity (Fig. 3). A contrast-enhanced computerized tomography (CT) scan of the nose and paranasal sinuses showed a mild contrast-enhancing mass arising from the lateral aspect of left nasal vestibule and filling the anterior aspect of nasal cavity. There was no extension beyond the nasal cavity into the nasopharynx or any paranasal sinuses. This made us not to concern about preoperative interventions to minimize intraoperative bleeding. The mass was excised with unipolar cautery (Fig. 4). It presented of hard consistency (Fig. 5) and intraoperative bleeding was minimal (Fig. 6). Unilateral anterior nasal packing was done (Fig. 7). Postoperative recovery was uneventful. The pack was removed after 48 hours and the patient was discharged without any complications. Histopathological examination revealed dilated, cavernous vascular spaces lined with endothelial cells and separated by fibrostroma with stromal cell nuclei (Fig. 8). These findings were compatible with a diagnosis of angiofibroma. At 6 months follow-up, anterior rhinoscopy showed a normal vestibule with no evidence of residual or recurrent disease (Fig. 9). 


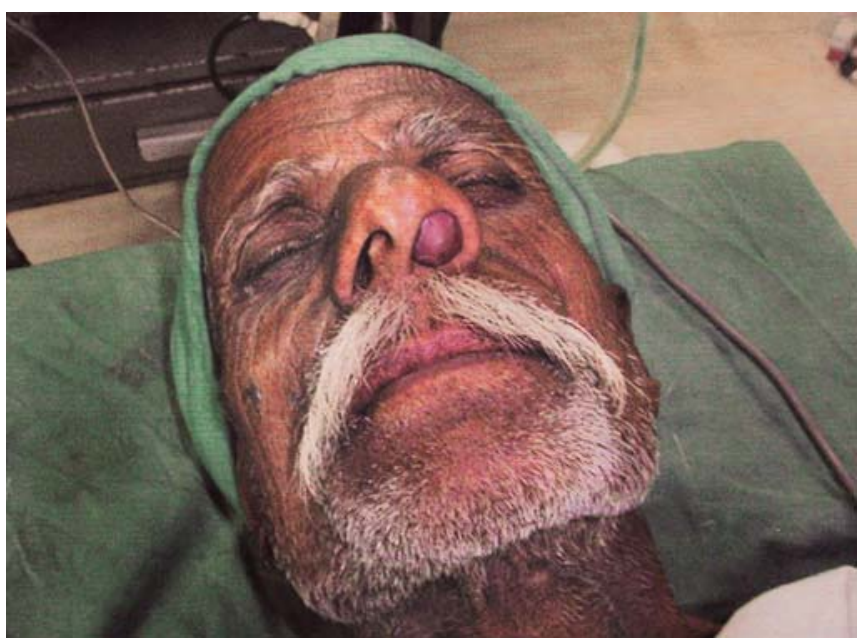

Fig. 1: Mass in left nasal cavity

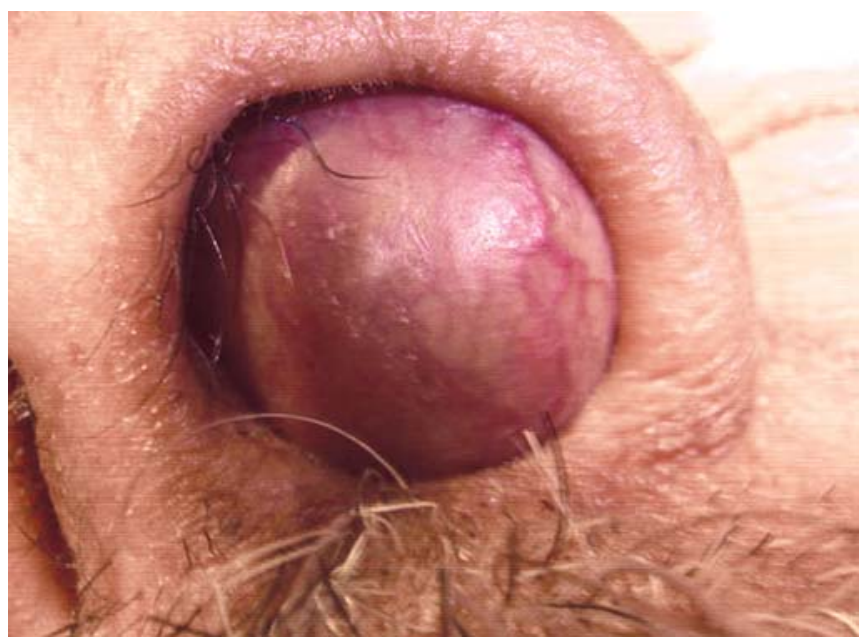

Fig. 2: Vessels over the mass

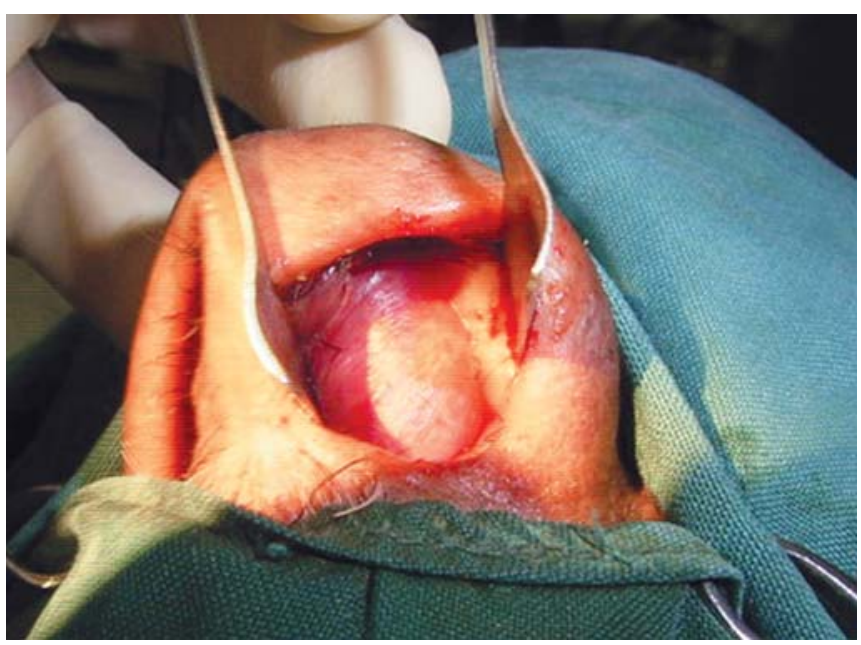

Fig. 3: Attachment to left nasal vestibule

\section{DISCUSSION}

Nasopharyngeal angiofibroma is a well-defined entity sharply localized in time, space and sex. The tumor virtually

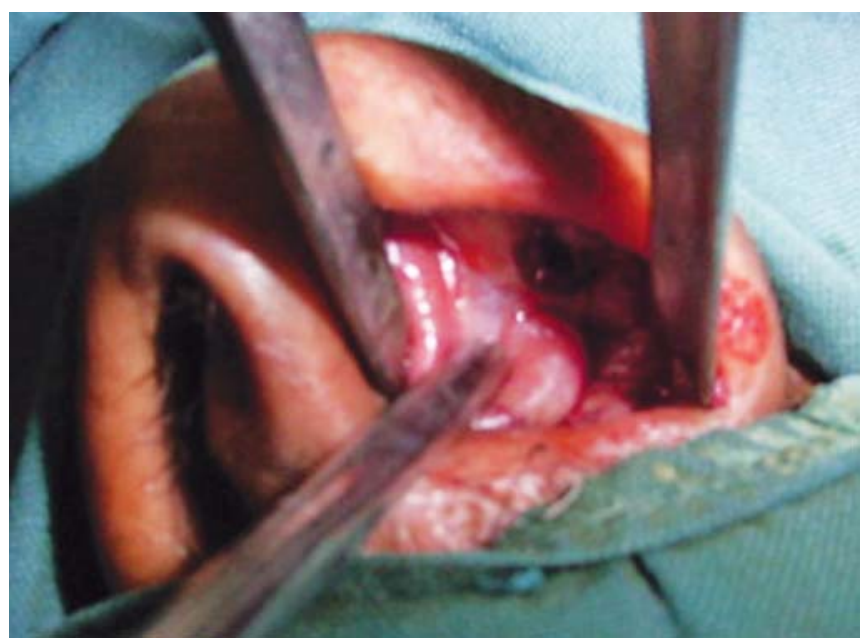

Fig. 4: Excision by unipolar cautery

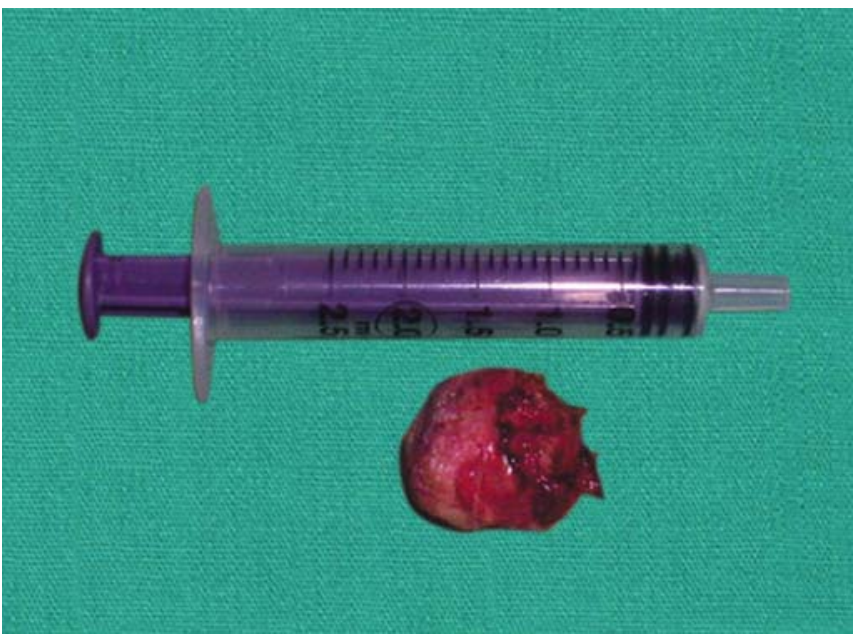

Fig. 5: Excised mass from left nasal vestibule

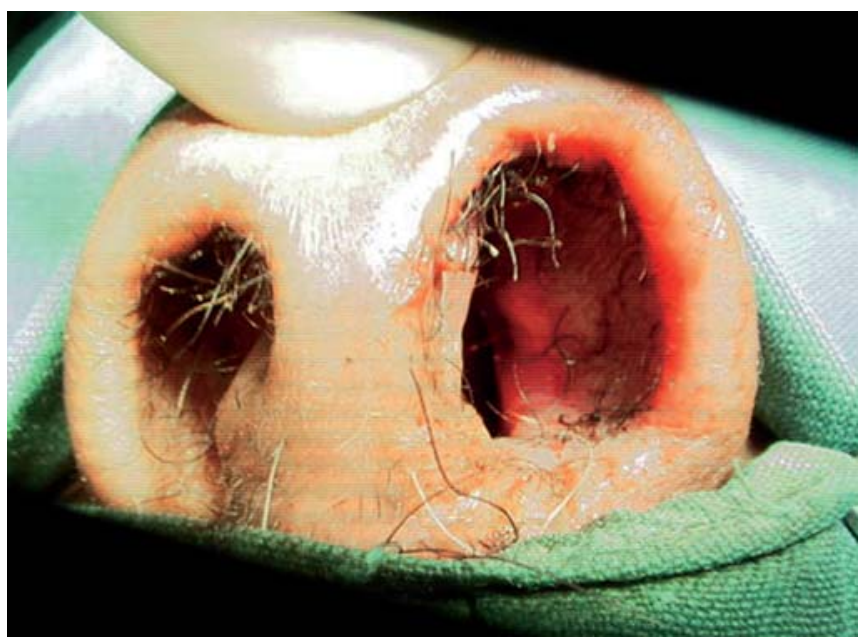

Fig. 6: Immediate postoperative raw area in left nasal vestibule

always arises from the nasopharynx and only later may extend into the nasal cavity. ${ }^{10}$ More recently, the term extranasopharyngeal angiofibroma has been applied to 


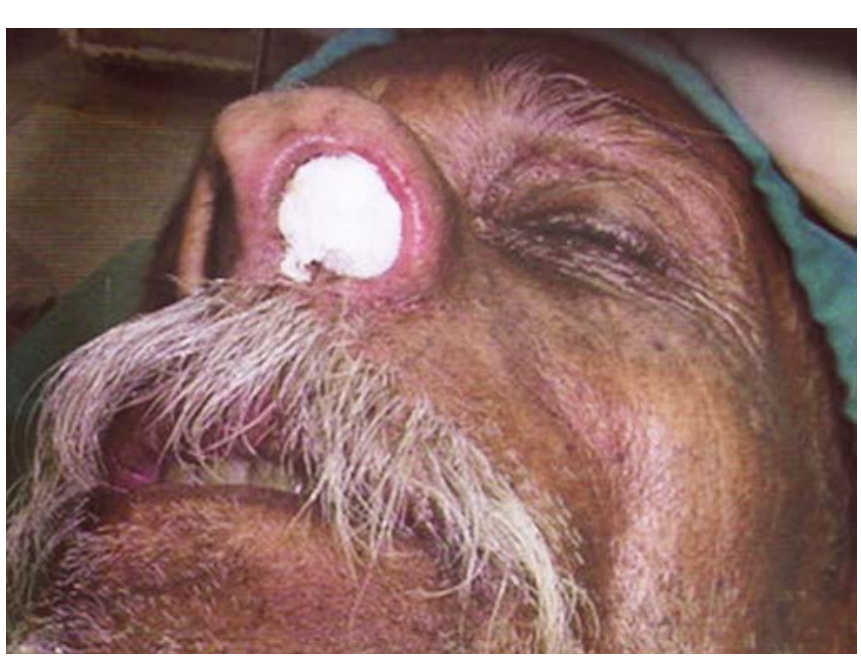

Fig. 7: Unilateral anterior nasal pack

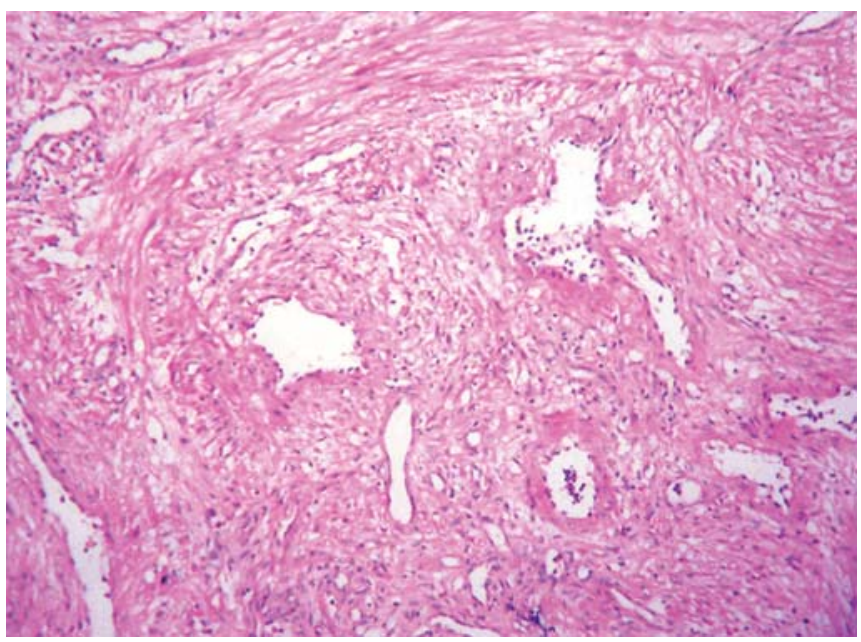

Fig. 8: Dilated cavernous vascular spaces lined by endothelium separated by fibrostroma with stromal cell nuclei

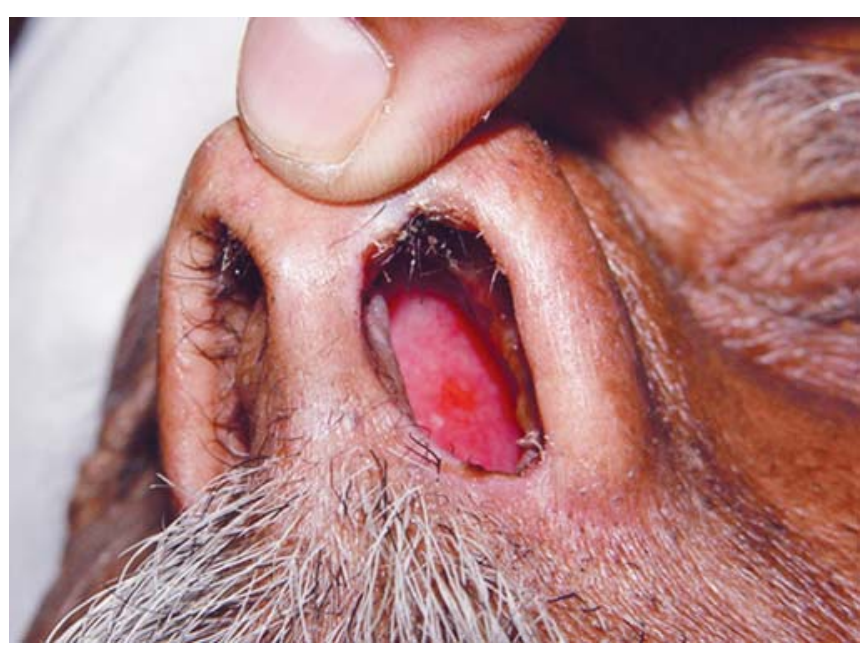

Fig. 9: Follow-up after 6 months normal left nasal vestibule

vascular, fibrous nodules occurring outside the nasopharynx. However, extranasopharyngeal angiofibroma have virtually nothing in common with nasopharyngeal tumors and the use of the term angiofibroma for these lesions may therefore be confusing. In fact, these rare benign neoplasms characterized by a different biological history and clinical features with respect to nasopharyngeal tumors and, for these reasons, they should be regarded as atypical clinical entity. Compared to nasopharyngeal angiofibromas, patients affected are older, symptoms develop more quickly, and hypervascularity is less common. ${ }^{5}$ The case described here fulfilled all these features. The clinical presentation of extranasopharyngeal angiofibroma depends mainly on the localization and extent of the tumor. In those cases, arising from nasal cavities, nasal obstruction and epistaxis are the more frequently reported symptoms. DNE, CT scan and magnetic resonance imaging (MRI) are used to determine the tumor site and its extension, with special attention being focused on skull-base involvement, intracranial spread and relationship to important vascular and neurologic structures. ${ }^{11}$ The administration of a contrast agent in nasopharyngeal angiofibroma leads to a strong and usually homogeneous enhancement on CT and MRI T1 sequences. ${ }^{12}$ On the other hand, extranasopharyngeal angiofibroma usually enhances contrast medium or even nothing, due to the frequent poor vascularity of the tumor. ${ }^{10}$ Alvi et al ${ }^{3}$ consider CT scan to be sufficient for the diagnosis of extranasopharyngeal angiofibroma, as it clearly delineates and identifies the tumor. In our case, the poor vascularity of the lesion did not require preoperative concerns to minimize intraoperative bleeding. Surgical excision of the mass is the treatment of choice, and recurrence is rare. ${ }^{2}$ Extranasopharyngeal angiofibroma must be taken into consideration in the differential diagnosis of nasal vascular tumors and the nasal vestibule should be regarded as a potential, though exceptional, localization of these neoplasms. Clinically, extranasopharyngeal angiofibromas, such as the one described in this case, fall into that category of atypical presentations that do not fit within the accepted clinical parameters that one always expects to find in cases of angiofibroma.

\section{SUMMARY}

- Extranasopharyngeal angiofibroma must be taken into consideration in the differential diagnosis of nasal vascular tumors.

- The nasal vestibule should be regarded as a potential, though exceptional, localization of these neoplasms.

- Clinically, extra nasopharyngeal angiofibromas, such as the one described in this case, fall into that category of atypical presentations that do not fit within the accepted clinical parameters that one always expects to find in cases of angiofibroma. 


\section{REFERENCES}

1. De Vincentiis G, Pinelli V. Rhinopharyngeal angiofibroma in the pediatric age group. Clinical-statistical contribution. Int J Pediatr Otorhinolaryngol 1980;2:99-122.

2. Sarpa JR, Novely NJ. Extranasopharyngeal angiofibroma. Otolaryngol Head Neck Surg 1989;101:693-697.

3. Alvi A, Myssiorek D, Fucks A. Extranasopharyngeal angiofibroma. J Otolaryngol 1996;25:346-348.

4. Huang RY, Damrose EJ, Blackwell KE, Cohen AN, Calcaterra TC. Extranasopharyngeal angiofibroma. Int J Pediatr Otorhinolaryngol 2000;561:59-64.

5. Akbas Y, Anadolu Y. Extranasopharyngeal angiofibroma of the head and neck in women. Am J Otolaryngol 2003;24:413-416.

6. Windfuhr JP, Remmert S. Extranasopharyngeal angiofibroma: etiology, incidence and management. Acta Otolaryngol 2004;124:880-889.

7. Somdas MA, Ketenci I, Unlu Y, Canoz O, Guney E. Extranasopharyngeal angiofibroma originating from the nasal septum. Otolaryngol Head Neck Surg 2005;133:1-2.

8. Lucas RB. Pathology of tumours of the oral tissues. Third ed Edinburgh: Churchill Livingstone 1976.
9. Celik B, Erisen L, Saraydaroglu O, Coskun H. Atypical angiofibromas: a report of four cases. Int J Pediat Otorhinolaryngol 2005;69:415-421.

10. Harrison DF. The natural history, pathogenesis, and treatment of juvenile angiofibroma. Personal experience with 44 patients. Arch Otolaryngol Head Neck Surg 1987;113:936-942.

11. Schick B, Kahle G. Radiological findings in angiofibroma. Acta Radiol 2000;41:585-593.

12. Maroldi R, Berlucchi M, Farina D, Tomenzoli D, Borghesi A, Pianta L. Benign neoplasm and tumor-like lesions. In: Maroldi $\mathrm{R}$, Nicolai P, editors. Imaging in treatment planning for sinonasal diseases. Berlin, Heidelberg, New York: Springer Verlag 2005. p. 107-158.

\section{ABOUT THE AUTHOR}

\section{Sharanabasappa Rudragouda Malipatil}

Associate Professor, Department of ENT and Head and Neck Surgery Shri BM Patil Medical College, BLDE University, Bijapur, Karnataka India, Phone: 9341083859, e-mail: sharnu_rmp@yahoo.co.uk 